\title{
Durable response in a thymic neuroendocrine tumor: A case report and review of the literature
}

\author{
Yusef A. Syed ${ }^{1}$, Daniel H. Miller*2, Liuyan Jiang ${ }^{3}$, Arya Bagherpour ${ }^{4}$, Stephen J. Ko ${ }^{5}$ \\ ${ }^{1}$ Jacobs School of Medicine and Biomedical Sciences, Buffalo, NY, United States \\ ${ }^{2}$ Department of Radiation Oncology, The Mayo Clinic, Jacksonville, FL, United States \\ ${ }^{3}$ Department of Pathology, The Mayo Clinic, Jacksonville, FL, United States \\ ${ }^{4}$ Department of Radiology, The University of Florida, Jacksonville, FL, United States \\ ${ }^{5}$ Department of Radiation Oncology, The Mayo Clinic, Jacksonville, FL, United States
}

Received: January 20, 2017

DOI: $10.5430 /$ jst.v7n2p23
Accepted: April 5, 2017

Online Published: April 19, 2017

\begin{abstract}
Thymic neuroendocrine tumors are rare, representing less than 5\% of all thymic cancers, and are biologically distinct in histology and behavior. Conclusive treatment guidelines are limited due to a lack of randomized clinical trials. Current literature, consisting mainly of case reports and series, suggests that complete surgical resection combined with adjuvant radiation therapy offers the greatest survival benefit for patients with thymic neuroendocrine tumors (NET). Adjuvant chemotherapy may also delay the onset of distant metastasis. Prognosis is generally poor, particularly in high-grade tumors, as these patients are prone to early metastasis. Here we present the case of a 62-year-old woman who achieved a durable response after undergoing an aggressive multimodal treatment approach for a primary thymic NET that initially presented as chronic cough and mild dyspnea on exertion.
\end{abstract}

Key Words: Thymus, Neuroendocrine tumor, Intensity modulated radiation therapy, Chemoradiation

\section{INTRODUCTION}

Primary neuroendocrine carcinoma of the thymus is a relatively uncommon diagnosis, representing approximately five percent of all thymic cancers. ${ }^{[1]}$ In addition, there is an association with multiple endocrine neoplasia types 1 and 2, and Cushing's syndrome in an estimated $35 \%$ of cases. ${ }^{[2-4]}$ Historically, these tumors were designated as thymic carcinoids, but naming conventions have been questioned in recent years given the aggressive nature of this disease. There is considerable variability in the histologic features exhibited in these tumors that significantly impacts prognosis. ${ }^{[5]}$ Since no randomized clinical trials have been conducted to date, treatment recommendations are based upon case reports and practitioner experience. Here we present the case of an isolated primary thymic neuroendocrine tumor (NET) that responded favorably to a multimodal treatment strategy consisting of surgery and adjuvant chemoradiation, followed by consolidative chemotherapy.

\section{Case presentation}

A 62-year-old Caucasian woman presented to her primary physician with a chronic cough of fifteen-year duration that worsened over a period of months. At that time the cough was non-productive and the patient denied hemoptysis and chest pain, though she did acknowledge mild dyspnea on exertion and a sensation of "heaviness" in the chest, along

*Correspondence: Daniel H. Miller; Email: miller.daniel@ mayo.edu; Address: Department of Radiation Oncology, The Mayo Clinic, 4500 San Pablo Rd S., Jacksonville, FL 3224, United States. 
with recent intermittent episodes of diaphoresis. She was a former smoker with a 30 pack-year history, and had a family history of breast cancer (mother and sister) and prostate cancer (maternal grandfather). Her physical exam was unremarkable and she exhibited no Cushingoid features. Routine laboratory analysis was also entirely normal.

A chest $x$-ray performed at that time was read as abnormal and the patient subsequently underwent a high resolution contrast tomography (CT) scan of the chest. This scan confirmed the presence of a $4.1 \mathrm{~cm} \times 5.6 \mathrm{~cm}$ anterior mediastinal mass. She was then referred to cardiothoracic surgery and underwent en bloc resection of the mass. During the proce-
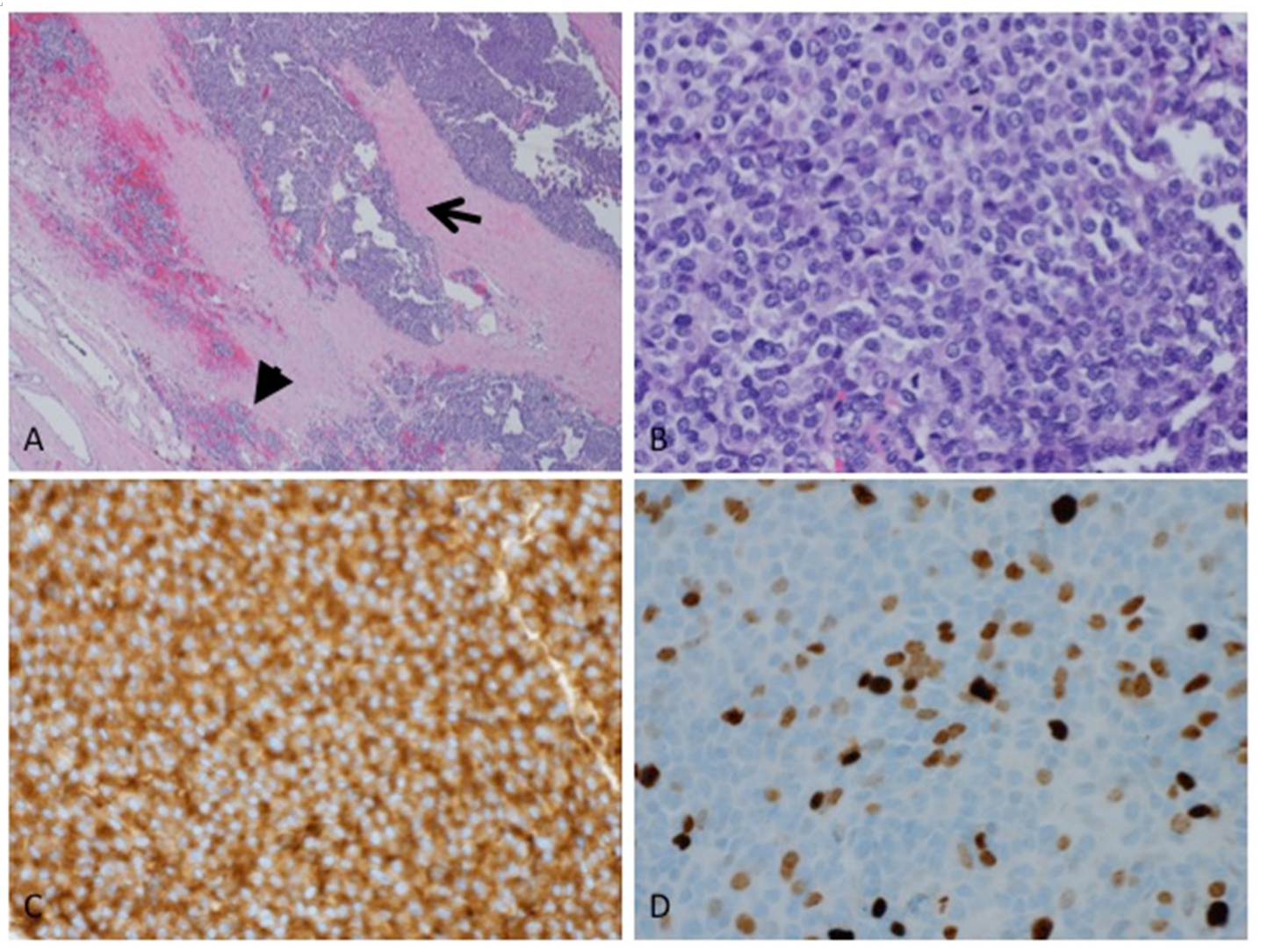

Figure 1. H\&E sections show the monotonous tumor cells with nesting and trabecular proliferation. The capsular invasion (arrow) and lymphovascular invasion (arrow head) were identified (A, H\&E $\times 4$ ). Mitoses are readily seen $(\mathrm{B}, \mathrm{H} \& \mathrm{E} \times 40)$. Immunochemical studies reveal the tumor cells to be positive for synaptophysin $(\mathrm{C}, \times 40)$, pancytokeratin, $\mathrm{CD} 56$, and chromogranin; the proliferative rate by Ki-67 $(\mathrm{D}, \times 40)$ is high $(20 \%-30 \%)$. The overall pathologic findings support the diagnosis of high-grade neuroendocrine carcinoma of the thymus.

Given the aggressive nature of NETs, a comprehensive diagnostic workup was conducted. Magnetic resonance imaging of the brain was negative for cranial metastasis. Positron emission tomography CT (PET-CT) [F-18]Fluorodeoxygludose (FDG) showed a mild asymmetric increase in uptake within the right thyroid gland, but no additional areas of concerning uptake. Notably the patient had dure, the mass was found to be extending into the parietal pleura, though overt extra-parietal invasion was noted. The surgeon observed an abnormal nodule in the right lower lobe of the lung, prompting an anterior wedge resection of that lobe. Surgical pathology examination rendered a diagnosis of high-grade neuroendocrine carcinoma of the thymus with capsular and lymphovascular invasion, measuring $6.5 \mathrm{~cm} \times$ $6.0 \mathrm{~cm} \times 4.0 \mathrm{~cm}$. Immunostaining revealed the tumor cells to be positive for CD56, synaptophysin, pancytokeratin, and chromogranin. Ki-67 was elevated at $20 \%-30 \%$, indicating a high rate of mitosis, as highlighted in Figure 1. The lung specimen was found to be normal parenchyma with a benign $0.4 \mathrm{~cm}$ intraparenchymal lymph node. 
$\left(60 \mathrm{mg} / \mathrm{m}^{2}\right)$ on day 1 and etoposide $\left(120 \mathrm{mg} / \mathrm{m}^{2}\right)$ on days 1 to 3 , with the intention of completing four cycles. After the first cycle she developed grade 2 neutropenia, grade 2 anemia, fever, and mild bilateral tinnitus. Her doses of both chemotherapy agents were reduced by twenty percent and she was placed on a seven day course of oral levofloxacin, after which the fever resolved. She experienced moderate persistent nausea with episodes of vomiting early in the course of her treatment. These symptoms were controlled with a combination of prochlorperazine and ondansetron that was later augmented with aprepitant. Given her history of chemotherapy-induced neutropenia, the patient was placed on pegfilgrastim on day 4 of chemotherapy cycles three and four. She tolerated cycles two and three well, and was administered her original full doses of cisplatin and etoposide for cycle four. The final two cycles were administered after completion of her radiation therapy and are thus can be considered as consolidative.
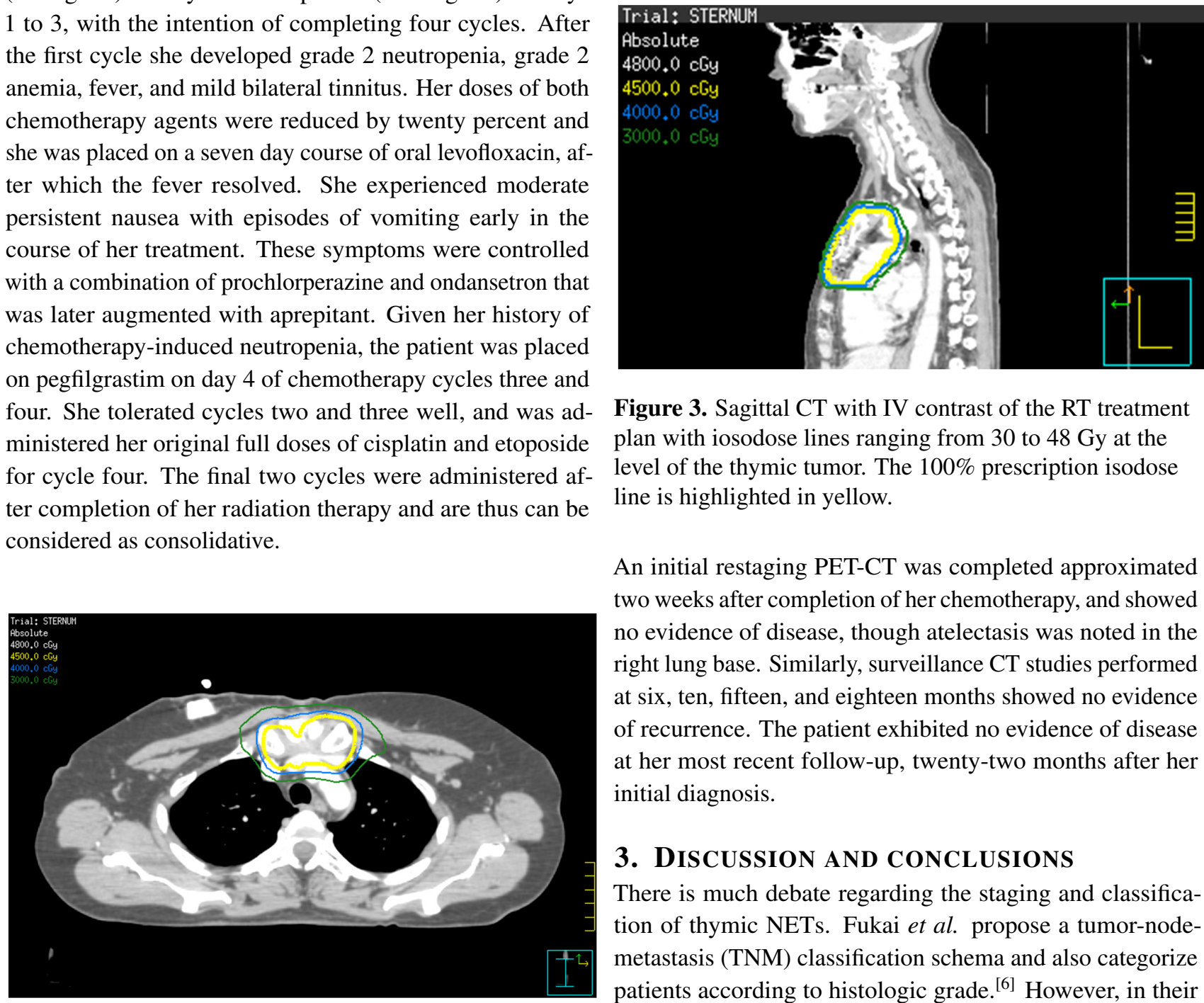

Figure 3. Sagittal CT with IV contrast of the RT treatment plan with iosodose lines ranging from 30 to $48 \mathrm{~Gy}$ at the level of the thymic tumor. The $100 \%$ prescription isodose line is highlighted in yellow.

An initial restaging PET-CT was completed approximated two weeks after completion of her chemotherapy, and showed no evidence of disease, though atelectasis was noted in the right lung base. Similarly, surveillance CT studies performed at six, ten, fifteen, and eighteen months showed no evidence of recurrence. The patient exhibited no evidence of disease at her most recent follow-up, twenty-two months after her initial diagnosis.

\section{DisCUSSION AND CONCLUSIONS}

There is much debate regarding the staging and classification of thymic NETs. Fukai et al. propose a tumor-nodemetastasis (TNM) classification schema and also categorize patients according to histologic grade. ${ }^{[6]}$ However, in their case series, neither tumor-node classification nor histologic

Figure 2. Axial CT chest with IV contrast of the RT treatment plan with iosodose lines ranging from 30 to $48 \mathrm{~Gy}$ at the level of the resected thymic tumor in the upper anterior mediastinum. The $100 \%$ prescription isodose line is highlighted in yellow.

Her course of radiation therapy consisted of a total of thirty 1.5 Gray (Gy) fractions delivered twice daily for a total of 45 Gy to the surgical bed. The radiation therapy (RT) was delivered using an image-guided intensity modulated RT technique. Figures 2 and 3 display the treatment plan and identify various isodose lines ranging from 30-48 Gy. She tolerated the RT well, with the only side effect being mild erythema of the anterior chest treated with topical lotion. She did, however, report worsening of her chronic cough and was started on hydrocodone bitartrate and homatropine methylbromide (Hycodan) syrup. Her cough gradually improved upon completion of chemoradiation and the Hycodan syrup was discontinued. grade reliably predicted prognosis. Factors that did appear to influence outcomes were the presence of Cushing's syndrome, extracapsular extension, and the development of distant metastases, all of which portend poor prognosis. Tiffet et al. categorize patients according to post-surgical staging and also find that survival is not linked to histologic grade or the immunohistochemical staining profile of individual tumors. ${ }^{[7]}$ Median survival ranged from 82 months for stage I disease to 27 months for stage IVA disease. Conversely, Moran and Suster did find an inverse correlation between degree of differentiation and disease-free survival in the eighty patients included in their case series. ${ }^{[5]}$ Five year survival fell from fifty percent for well-differentiated tumors to zero for poorly-differentiated ones. In addition, the authors assert that mediastinal NETs are biologically distinct as compared to their foregut (i.e. pulmonary) counterparts and result in comparatively lower overall survival at 5 and 10-year follow-up. This case represents a high-grade neuroendocrine carcinoma 
of the thymus according to the grading system described by Drs. Moran and Suster.

Thymic NETs are best detected on contrast-enhanced CT, but adjunctive scintigraphic imaging modalities may be utilized for surveillance imaging and detection of metastatic disease. [F-18]- FDG PET/CT is a useful tool in detecting thymic NETs, particularly in patients with aggressive tumors, but may be limited in detecting indolent disease. Alternative radiopharmaceuticals should be considered when [F-18]- FDG PET scans are negative, including Indium-111 pentetreotide (Octreoscan), [Ga-68]- DOTA-TOC, and I-123 MIBG. ${ }^{[8]}$ Indium-111 pentetreotide (Octreoscan) is useful in detecting somatostatin receptors, which are often expressed in neuroendocrine tumors, and it may be helpful in detecting thymic NETs that are PET negative. ${ }^{[9]}$ More recently, the use of [Ga-68]- DOTA-peptide in patients with NETs has shown great promise, as this radiopharmaceutical may have higher diagnostic performance in this patient population. ${ }^{[10]}$

Radical surgical resection by means of median sternotomy is the mainstay of treatment. ${ }^{[1,11]}$ Adjuvant RT, often combined with concurrent or consolidative chemotherapy, is common in this setting though the survival benefits of adjuvant therapy have yet to be quantified due to a lack of randomized clinical trials. However, Tiffet et al. note an apparent benefit to radiation therapy in terms of local control. Six of eleven patients who underwent surgical resection in their case series relapsed locally, five of whom received no RT. Fukai et al. treated eight of their fifteen patients with post-op RT and conclude such treatment "might result in increased survival", while Wick et al. unequivocally recommend adjuvant RT in

\section{REFERENCES}

[1] Oberg K, Hellman P, Ferolla P, et al. Neuroendocrine bronchial and thymic tumors: ESMO Clinical Practice Guidelines for diagnosis, treatment and follow-up. Ann Oncol. 2012; 23 Suppl 7: vii120-3. https://doi.org/10.1093/annonc/mds267

[2] Lausi PO, Refai M, Filosso PL, et al. Thymic neuroendocrine tumors. Thorac Surg Clin. 2014; 24: 327-32. PMid:25065934. https ://doi.org/10.1016/j.thorsurg. 2014.05.007

[3] Gaude GS, Hattiholi V, Malur PR, et al. Primary neuroendocrine carcinoma of the thymus. Niger Med J. 2013; 54: 68-71. PMid:23661903. https ://doi.org/10.4103/0300-1652.1089 04

[4] Wick MR, Rosai J. Neuroendocrine neoplasms of the thymus. Pathol Res Pract. 1988; 183: 188-99. https ://doi.org/10.1016/S034 4-0338(88) 80046-3

[5] Moran CA, Suster S. Neuroendocrine carcinomas (carcinoid tumor) of the thymus. A clinicopathologic analysis of 80 cases. Am J Clin Pathol. 2000; 114: 100-10. PMid:10884805. https://doi.org/ 10.1309/3PDN-PMT5-EQTM-HOCD an earlier series of seven patients. ${ }^{[12]}$ Distant metastases are common, with various case series reporting frequencies of between fifty and ninety-two percent. ${ }^{[13]}$ Given this, adjuvant chemotherapy is often administered and Cisplatin-based regimens are the favored approach. In addition, the European Society for Medical Oncology (ESMO) notes that temozolomide-based treatments may offer some benefit. Furthermore, somatostatin analogs can be used to ameliorate the symptoms of Cushing's syndrome, should they arise. Surgical resection for local and distant recurrence should be performed when feasible as empirical evidence points to a survival benefit. ${ }^{[6,7]}$ Finally, ESMO guidelines specifically recommend RT for brain and bone metastases.

In conclusion, achieving a durable response in patients with thymic NETs is difficult. The patient presented here underwent an aggressive multimodal treatment approach consisting of an R0 resection, concurrent adjuvant chemoradiation, and consolidative chemotherapy. She did have a favorable risk profile, as her tumor exhibited no extra-capsular extension and she remained free of Cushing's symptoms throughout the course of her disease. The treatment of high grade thymic NETs remains a significant clinical challenge, and the field must endeavor to address shortcomings in our knowledge base by conducting randomized clinical trials, specifically evaluating the survival benefits of pre- and/or post-op RT. Furthermore, the role of post-op chemotherapy in the control of distant recurrence must be further elucidated.

\section{CONFlicts of InTERest Disclosure}

The authors declare that there is no conflict of interest statement.

[6] Fukai I, Masaoka A, Fujii Y, et al. Thymic neuroendocrine tumor (thymic carcinoid): a clinicopathologic study in 15 patients. Ann Thorac Surg. 1999; 67: 208-11. https://doi.org/10.1016/S0 003-4975 (98) 01063-7

[7] Tiffet O, Nicholson AG, Ladas G, et al. A clinicopathologic study of 12 neuroendocrine tumors arising in the thymus. Chest. 2003; 124 141-6. PMid:12853516. https://doi.org/10.1378/chest.12 4.1.141

[8] Groves AM, Mohan HK, Wegner EA, et al. Positron emission tomography with FDG to show thymic carcinoid. AJR Am J Roentgenol. 2004; 182: 511-3. PMid:14736691. https://doi.org/10.2214/ ajr.182.2.1820511

[9] Borrero CG, McCook B, Mountz JM. Indium-111 pentetreotide imaging of carcinoid tumor of the thymus. Clin Nucl Med. 2005; 30: 218-21. PMid:15764874. https://doi.org/10.1097/01.rlu.0 000156799.23492 .54

[10] Ito T, Jensen RT. Molecular imaging in neuroendocrine tumors: recent advances, controversies, unresolved issues, and roles in management. Curr Opin Endocrinol Diabetes Obes. 2017; 24: 15-24. PMid:27875420. 
[11] Rena O, Filosso PL, Maggi G, et al. Neuroendocrine tumors (carcinoid) of the thymic gland. Ann Thorac Surg. 2003; 75: 633. https://doi.org/10.1016/S0003-4975(02) 04329-1

[12] Wick MR, Scott RE, Li CY, et al. Carcinoid tumor of the thymus: A clinicopathologic report of seven cases with a review of the literature.
Mayo Clin Proc. 1980; 55: 246-54. PMid:6153740.

[13] de Montpreville VT, Macchiarini P, Dulmet E. Thymic neuroendocrine carcinoma (carcinoid): a clinicopathologic study of fourteen cases. J Thorac Cardiovasc Surg. 1996; 111: 134-41. https: //doi.org/10.1016/S0022-5223(96)70409-9 